\title{
A modified surgical technique to simplify the procedure of femoral stem implantation for an appropriate anteversion angle during endoprosthetic reconstruction
}

\author{
Lijun Shi ${ }^{1 \#}$, Jun $\mathrm{Han}^{2 \#}$, Lili Shi ${ }^{3}$, Wei Sun ${ }^{1,4}$, Fuqiang Gao ${ }^{4}$ \\ ${ }^{1}$ Department of Orthopedics, Graduate School of Peking Union Medical College, China-Japan Friendship Institute of Clinical Medicine, Beijing, \\ China; ${ }^{2}$ Department of Spine Surgery, The First Affiliated Hospital of Dalian Medical University, Dalian, China; ${ }^{3}$ Department of Gastroenterology, \\ Henan Provincial People Hospital, Zhengzhou, China; ${ }^{4}$ Centre for Osteonecrosis and Joint-Preserving \& Reconstruction, Orthopaedic Department, \\ China-Japan Friendship Hospital, Graduate School of Peking Union Medical College, Beijing, China \\ Contributions: (I) Conception and design: W Sun; (II) Administrative support: All authors; (III) Provision of study materials or patients: All authors; \\ (IV) Collection and assembly of data: All authors; (V) Data analysis and interpretation: L Shi, J Han; (VI) Manuscript writing: All authors; (VII) Final \\ approval of manuscript: All authors. \\ "These authors contributed equally to this work. \\ Correspondence to: Prof. Wei Sun. Centre for Osteonecrosis and Joint-Preserving \& Reconstruction, Orthopaedic Department, China-Japan \\ Friendship Hospital, Graduate School of Peking Union Medical College, Chaoyang District, Beijing, China. Email: sun887@163.com.
}

Background In patients with bone tumors of the proximal femur, endoprosthetic reconstruction can provide stable and durable biological reconstruction. However, it is difficult and time-consuming to accurately place the femoral component for an appropriate anteversion angle after large tumor resection. We propose a modified surgical technique for simplifying the implantation process and promoting the stability of the artificial joint.

Methods: We retrospectively analyzed the clinical data of 28 patients undergoing endoprosthetic reconstruction at our center between 2009 and 2016. We used a traditional method (group A, n=11) and a modified surgical technique (group $\mathrm{B}, \mathrm{n}=17$ ) to determine the femoral anteversion angle during implantation of the femoral component. The modified surgical technique allowed more convenient and accurate implantation of the femoral components by externally rotating the shin at an angle of $15^{\circ}$. Surgical time and blood loss were designated as the primary outcomes, hospital stays, postoperative complications and functional recovery [Harris Hip Score (HHS); Musculoskeletal Tumor Society Score (MSTS)] were recorded as the secondary outcomes. The outcomes of the two groups were compared to verify the safety and efficacy of the modified surgical technique.

Results: The mean operative time was shorter in group B (109 \pm 10 minutes) than group A (131 \pm 6 minutes) $(\mathrm{P}<0.05)$. Although the average estimated intraoperative blood loss was less in group B $(814 \pm 35 \mathrm{~mL})$ than that in group $\mathrm{A}(839 \pm 30 \mathrm{~mL})$, there was no significant difference between the two groups $(\mathrm{P}=0.06)$. There was no significant difference between the two groups in hospital stays, postoperative complications, HHS or MSTS.

Conclusions: This modified surgical technique can simplify the process of femoral component implantation and significantly shorten operative time without increasing postoperative complications.

Keywords: Bone tumors; proximal femur; endoprosthetic reconstruction; anteversion; surgical technique

Submitted Apr 06, 2020. Accepted for publication Sep 30, 2020.

doi: $10.21037 / \mathrm{apm}-20-895$

View this article at: http://dx.doi.org/10.21037/apm-20-895 


\section{Introduction}

The proximal femur is the predilection site of primary benign and malignant bone tumors, and it is also a common site of metastases from different organs such as the breast, lung, prostate, and kidney (1). Regarding treatment options for this disease, surgery is often regarded as the preferred therapy for selected patients, which can effectively ease pain, relieve nerve and vascular compression, improve range of motion of the joint and reduce the risk of malignant transformation $(2,3)$. In past decades, amputation was usually used to save life, but would lead to an inability to walk following this operation. After resection of bone tumors, limb salvage surgery, including plate fixation $(4,5)$, intramedullary fixation (5-7) and endoprosthetic reconstruction (8-10), have gradually replaced amputation. Among these procedures, endoprosthetic replacement has become widely available in clinical practice and easily accepted by patients, allowing for adequate tumor excision and providing stable reconstruction for rapid functional recovery with early mobilization (11-13). The type of hip prosthesis can be divided into custommade prostheses, modular prostheses, or allograft-prosthesis composites $(14,15)$.

Despite these advantages of endoprosthetic replacement, this surgery is not perfect. There are still some technical difficulties in achieving mechanical stability of the prosthesis during this procedure, and the implant failure rate is up to $24.5 \%$ according to one study (16). Among common surgical failures of proximal femoral tumors, dislocation rates of $10.9 \%$ and $4.6 \%$ have been reported in the hemiarthroplasty and endoprosthetic reconstruction process, respectively $(17,18)$. One of the critical factors affecting stability of the hip joint is the correct positioning of the prosthesis, which is also necessary for an excellent joint range of motion. Additionally, accuracy of femoral stem anteversion angle would ensure the matching of the femoral head in the acetabular cup without impingement of the two components throughout the full range of body positions (19). Therefore, it is necessary to emphasize the anteversion angle of the femoral component during the implantation procedure.

In normal individuals, the femoral anteversion angle refers to the angle between the femoral neck axis and the posterior femoral condyle plane (20). So far, there is no standard method of accurately placing the prosthetic stem after osteotomy. Most surgeons rely on intraoperative visible or palpable anatomic landmarks and the anatomic morphology of the proximal femoral medullary cavity to position the stem within an intended target area. However, in patients with bone tumors of the proximal femur, there will be a significant segmental long bone block defect and loss of anatomic landmarks after extensive resection of large tumors, making it difficult to recognize the threedimensional geometry of the medullary cavity and achieve ideal anteversion of the femoral component. Traditionally, the method we often used to control the anteversion was to find the longest axis of the osteotomy cross-section of the femoral cavity after femoral osteotomy, then implant the femoral component at an angle of $5^{\circ}$ external rotation based on the longest axis. However, it is hard to determine the longest axis of the osteotomy cross-section of the femoral medullary cavity in one try, and usually requires additional time to repeatedly modify the angle.

To simplify the procedure of femoral stem implantation and improve the veracity and efficiency, we modified our traditional method through constant clinical practice. The purpose of this study was to illustrate the key aspects of this surgical technique and evaluate its clinical efficacy. We present the following article in accordance with the STROBE reporting checklist (available at http://dx.doi. org/10.21037/apm-20-895).

\section{Methods}

\section{Patients and study design}

This study was a retrospective cohort study investigating the effect of a new modified technique of reconstruction following resection of proximal femoral tumors. We retrospectively gathered information of all patients with bone tumors of the proximal femur from November 2009 through August 2016 by searching the electronic medical record of the China-Japan Friendship Hospital. We diagnosed bone tumors of the proximal femur according to the Clinical Practice Guidelines in Oncology for Bone Cancer (21). All participants gave informed consent prior to participation, and the study conformed to the provisions of the Declaration of Helsinki (as revised in 2013). This study was approved by the Ethics Committee of China-Japan Friendship Hospital (2020-QGW-108).

In this study, we included all patients who underwent this surgery for lesions in the upper one-third of the femur with an excision length of the lesion of at least $14 \mathrm{~cm}$, designating the knee joint and the top of the greater trochanter as reference points for the measurement. The pathological diagnosis included primary and metastatic bone tumors. 
Exclusion criteria were (I) lesions only confined to the femoral head without resection of the greater trochanter, (II) patients who were lost to follow-up. We included patients undergoing traditional surgery in group A, while the patients undergoing surgery by the modified technique were included in group B.

Based on the electronic medical record, we collected patients' medical information: demographic characteristics (sex, age), length of osteotomy, pathological type of tumor, surgical time, blood loss, hospital stay, complications, Harris Hip Score (HHS) and Musculoskeletal Tumor Society Score (MSTS) at follow-up times of 6,12 and 24 months. To compare the efficiency of the two surgical methods, we compared surgery time, blood loss, hospital stay, complications, HHS and MSTS between group A and group B. Because this modified surgical technique was designed to improve the complexity of the surgical procedure and reduce the surgery time, we designated surgical time and blood loss as the primary outcomes and other outcomes as the secondary outcomes.

\section{Surgical procedures of the traditional technique and modified technique}

For patients of both groups, this surgery typically involves extensive resection of the tumor lesion and reconstruction with cemented fixation of the femoral components. Indications for this surgery were (I) an impending bone fracture caused by cancer-associated osteolysis or a preexisting pathologic fracture; (II) intractable pain and functional disability; (III) effective control of the primary tumor. Contraindications to surgery included (I) estimated survival of fewer than 12 weeks; and (II) poor physical condition to undergo the procedure.

All included patients were treated by hemiarthroplasty, and surgeries were performed under general anesthesia via an extended posterolateral approach to the hip using a length-cemented stem (Chunli Zhengda, Beijing, China). Based on MRI and CT results, we defined the borderline and the resection range of the tumor preoperatively and then selected appropriately-sized femoral components. The incision was proximal to the greater trochanter, typically 15 to $25 \mathrm{~cm}$, in line with the long axis of the femur. The surgical area was fully exposed, the femur resection length was determined, and the tumor was excised with a negative surgical margin, ensuring that the plane of osteotomy was at least $3 \mathrm{~cm}$ away from the tumor lesion. After complete tumor excision, the femoral canal was reamed, and the test prosthesis was installed with an anteversion angle of approximately $15^{\circ}$, then the range of motion and stability of the joint were evaluated. After removing the test prosthesis, the marrow cavity was washed, bone cement was injected, and the final femoral stem prosthesis was implanted. Two typical cases are presented in Figures 1,2.

In group A patients $(n=11)$, we used the traditional method as described above to determine the anteversion angle during the implantation procedure. In group B patients $(n=17)$, the installation process for the femoral prosthesis was modified. The new surgical technique is shown in Figure 3 and was performed as follows: the patient was laid on the healthy side, the contralateral affected shin was straightened vertically upward with knee flexion of about $90^{\circ}$, keeping the sole of the foot upright and parallel to the horizontal plane; then the shin was externally rotated at an angle of $15^{\circ}$ by visual inspection, i.e., there was an approximate $15^{\circ}$ angle between the shin axis and the vertical line (red angle in Figure 3); at this angle, the axis of the femoral prothesis and the horizontal plane were completely coincident. The femoral components were then implanted horizontally. Figure 4 shows a schematic diagram of the diverse position of the femur.

\section{Postoperative treatment}

Postoperatively, all patients received prophylactic intravenous antibiotic treatment for 3 days. Prophylactic anticoagulation against deep venous thrombosis consisted of oral anticoagulant (rivaroxaban $10 \mathrm{mg}$ ) and anti-embolic stockings. Early postoperative functional exercise was performed by all patients from the lower limb isometric exercises when the anesthesia subsided to the leg and hip exercises. One month later, patients with malignant tumors were subjected to regular chemotherapy.

\section{Clinical efficacy assessment}

Clinical data on the surgery time and intraoperative blood loss were collected retrospectively from the medical records, and postoperative complications were also recorded in the follow-up. In the first 6 months, 12 months, and at each year thereafter, all patients were followed up to assess limb function recovery using the HHS (22) and MSTS (23). The duration of follow-up was calculated from the date of surgery to the date of the last visit. 

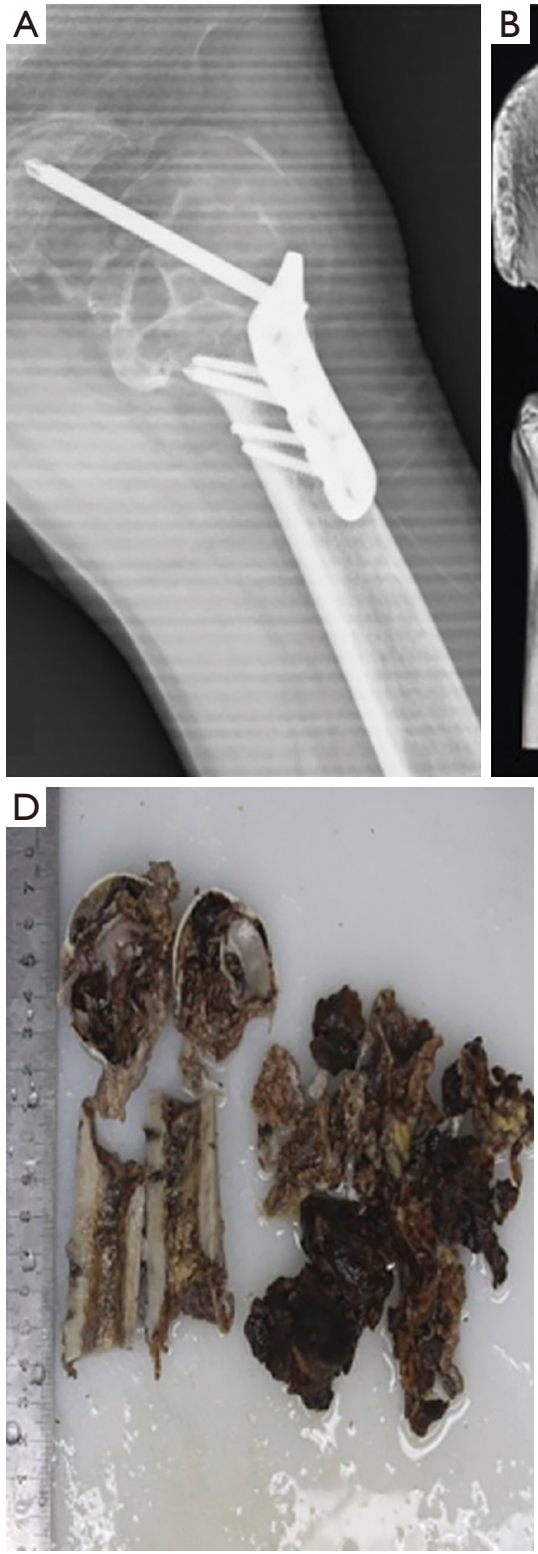
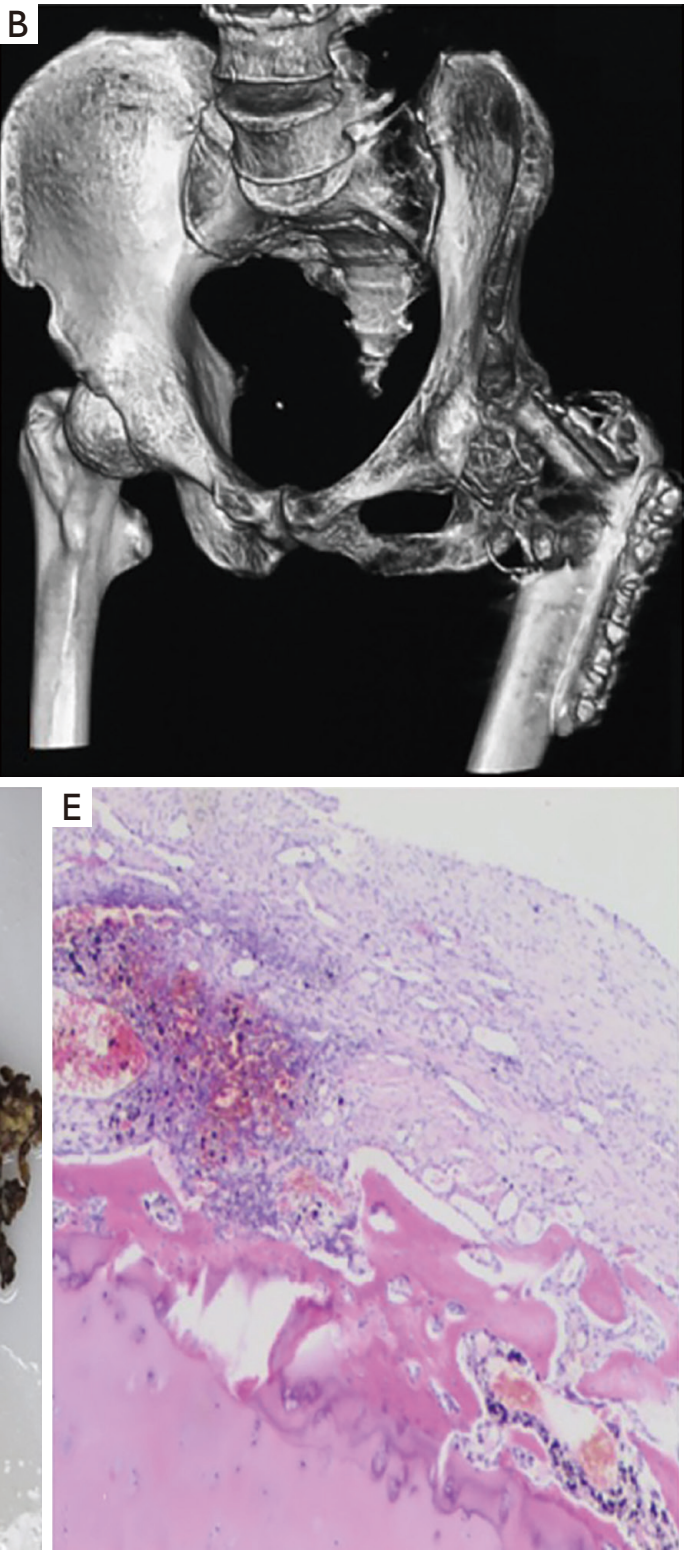
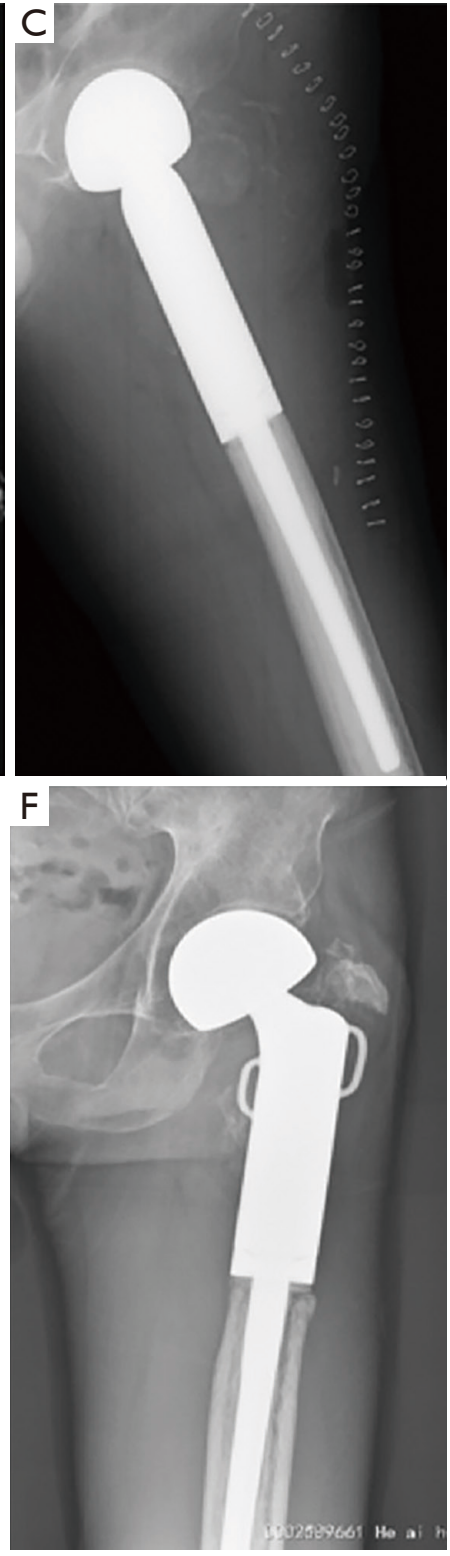

Figure 1 A 53-year-old male patient, who was diagnosed with an aneurysmal bone cyst in the left proximal femur and treated using a dynamic hip screw in 2006. He returned to our hospital complaining of severe left hip pain in May 2013. An anteroposterior radiograph (A), screw CT three-dimensional reconstruction (B) and other imaging tests of the pelvis showed severe bone erosion and pathological fracture of the hip joint. The patient was treated with hemiarthroplasty using a long-stem prosthesis (C), and resection specimen (D) was confirmed the recurrence of the aneurysmal bone cyst through pathological examination $(\mathrm{E}, \mathrm{H} \& \mathrm{E}$ stain; magnification, $\times 20)$. At the time of regular follow-up in October 2016, no radiographic evidence of loosening or dislocation was noted, except for heterotopic ossification around the hip joint visualized by anteroposterior radiograph of the pelvis $(\mathrm{F})$.

\section{Statistical analysis}

Data were analyzed using SPSS Statistical Software version 19.0 (IBM SPSS Statistics for Windows, Armonk, NY, USA). Quantitative variables are represented as the mean \pm standard deviation (SD). Differences in the measurement data were compared by $t$-test between the two groups, and count data were compared by the $\chi^{2}$ test. The level set for significance was set at $\mathrm{P}<0.05$. 

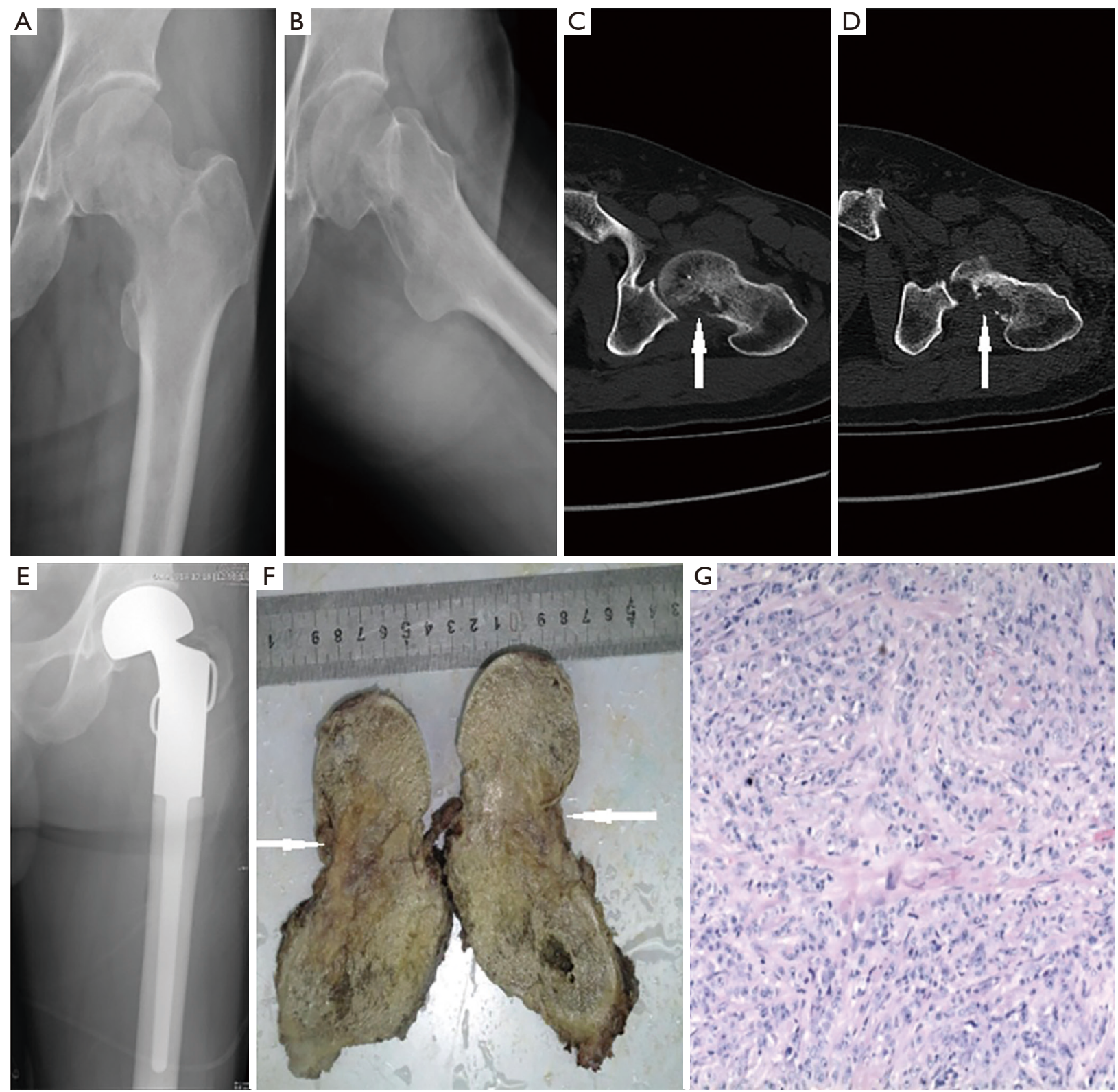

Figure 2 A 55-year-old male patient, who came to our hospital in September 2013 complaining of left hip pain and discomfort over the past 10 months. Anteroposterior (A) and frog leg lateral plain films (B) of both hips demonstrated that the thickness of the cortical bone in the left femoral neck had become thinner, and CT scans (C,D) showed bone destruction (white arrows) in the left femoral head and neck, which accords with the manifestations of bone tumors. However, no primary tumor location could be found by thorough examination. The patient received treatment involving hemiarthroplasty using a long-stem prosthesis (E), and resection specimen (F) was finally diagnosed with "metastatic low-differentiated adenocarcinoma of bone" through pathological examination (G, H\&E stain; magnification, $\times 100)$ and immunohistochemical analysis. Two years later, he was diagnosed with colon cancer accompanied by multiple lung metastases, and he died in 2016.

\section{Results}

From 2009 to 2016, we investigated a total of 35 patients at our center with bone tumors of the proximal femur.
After screening and assessment for eligibility, seven patients were eliminated by exclusion criteria and 28 patients were available for review. Of these patients, seven came to the hospital with pathologic fractures. The general preoperative 


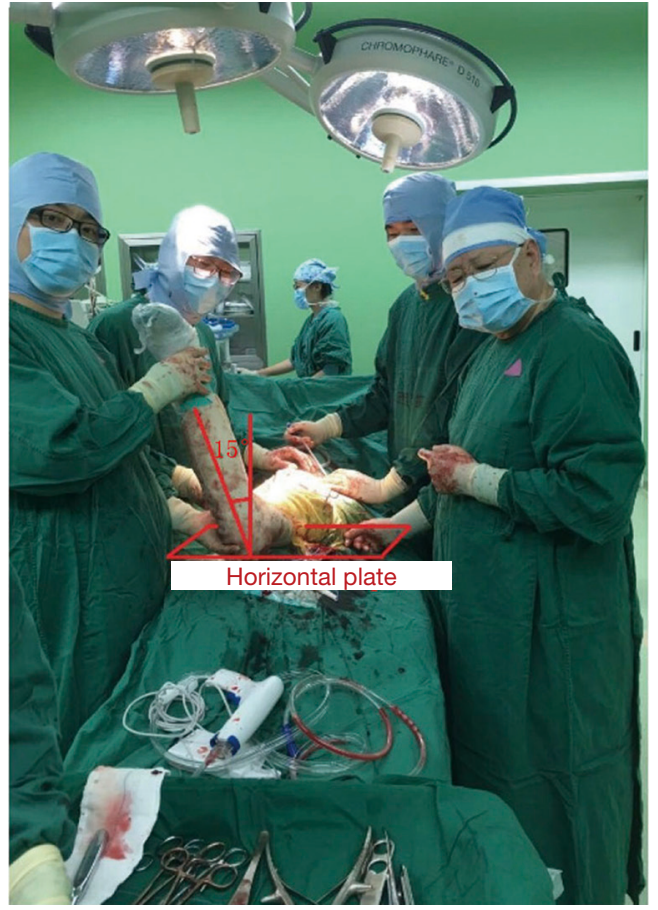

Figure 3 Surgical procedure: while maintaining this position, the knee joint is held and the ankle joint is rotated at an angle of approximately $15^{\circ}$ by visual inspection, then the medullary cavity is reamed and the femoral component implanted. conditions of both groups are listed in Table 1. All the 28 patients were treated with cemented hemiarthroplasty, and two approaches were used in the installation process of the femoral component to obtain an appropriate anteversion angle. The mean follow-up times in group A and group B were 39.8 (range, 32-55) and 40.4 (range, 34-51) months, respectively; three and six patients died from primary malignant tumors in group A and group B respectively during the follow-up period (Table 2).

Table 2 shows the general operation situation and postoperative complications of both groups. Compared to the traditional surgical method applied to group A patients (131.2 \pm 6.7 minutes), the new method takes an average of 21.9 minutes less in group B patients $(109.3 \pm 9.7$ minutes, $\mathrm{P}<0.01)$ for the complete procedure. There was no significant difference in the mean intraoperative blood loss between group A and group B patients $(839.1 \pm 30.6 v$ s. $814.5 \pm 35.3, \mathrm{P}=0.06$ ), although it tended to be less in group B patients (by a mean of $24.9 \mathrm{~mL}$ ). The mean length of hospital stay was similar in both groups of patients $(\mathrm{P}=0.38)$.

No significant complications occurred after surgery, and the complication rates were comparable in the two groups. Three patients in group A and two patients in group B experienced superficial wound infection at the time

A
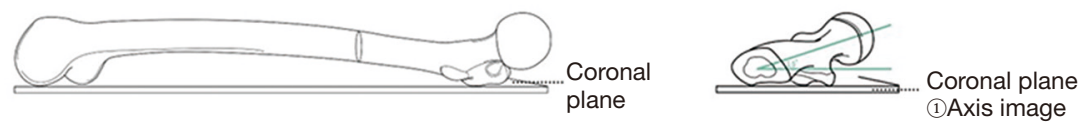

B
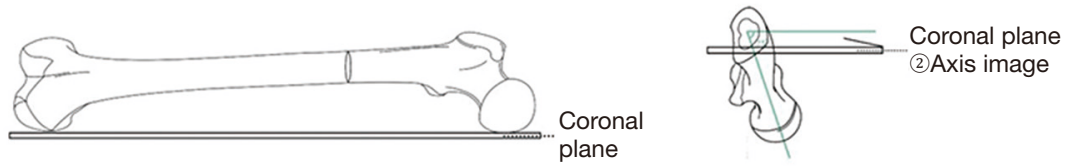

C
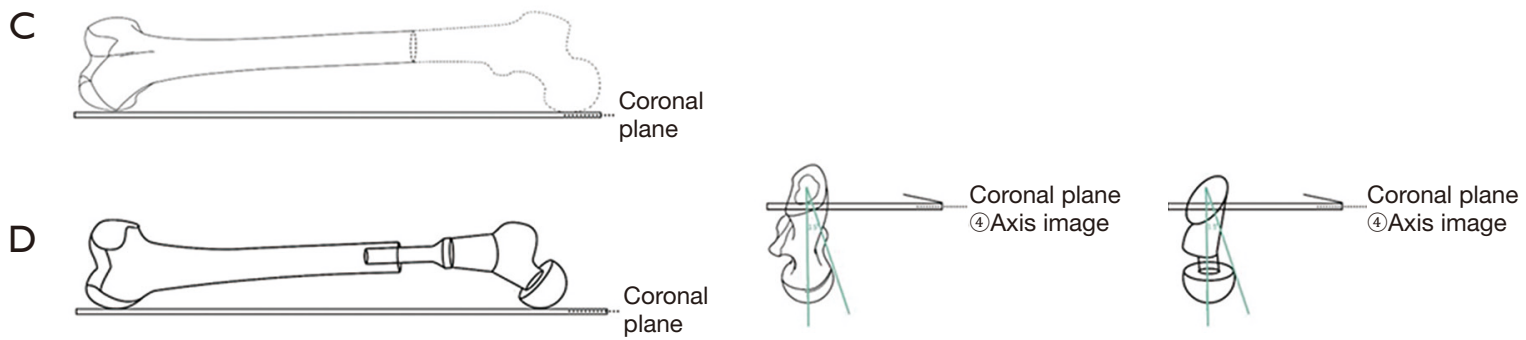

Figure 4 Schematic diagram of diverse positions of the femur. (A) Normal position of the femoral head; (B) femoral head dislocation after $90^{\circ}$ internal rotation of the femur; (C) excision of the proximal femur; (D) implantation of the femoral component horizontally after $15^{\circ}$ internal rotation of the femur. 
Table 1 Details of the general condition in the 28 patients

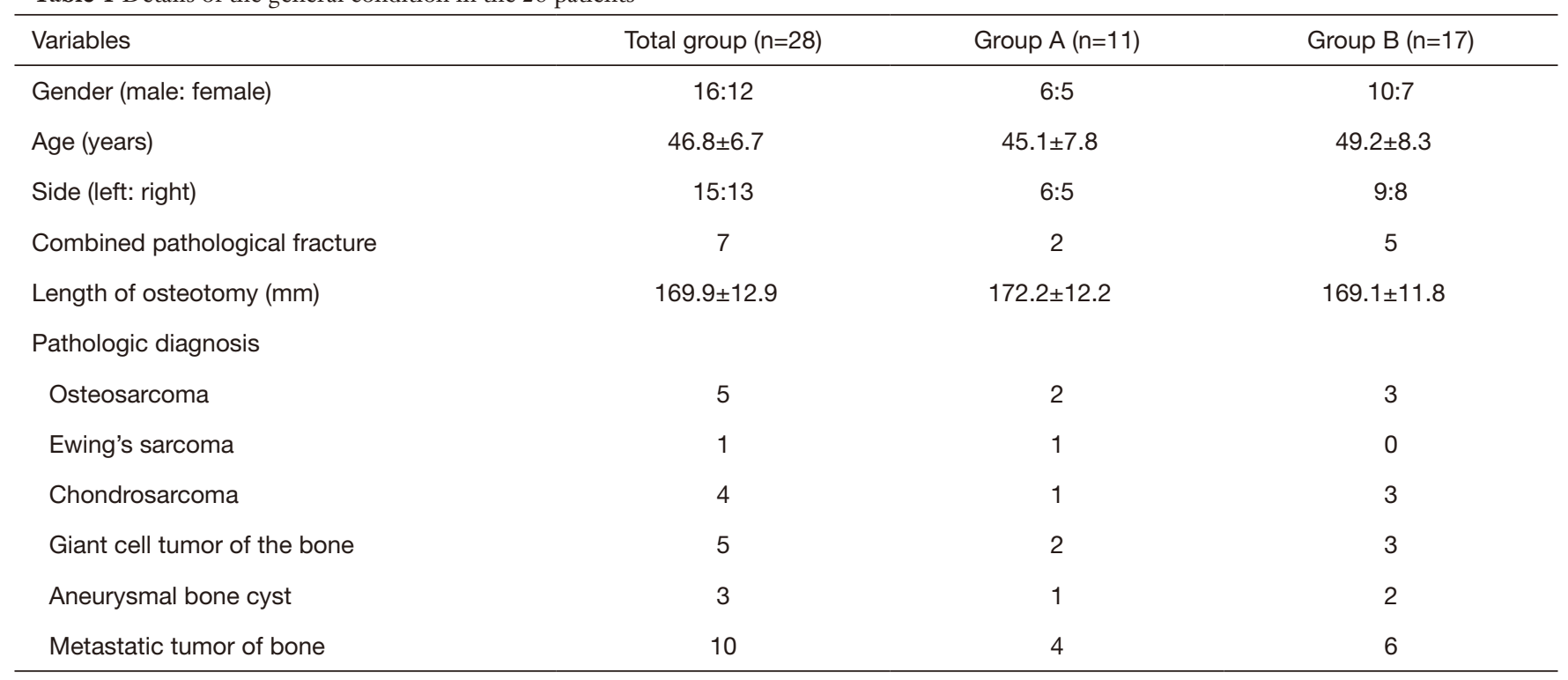

Table 2 The general operation situation and postoperative complications

\begin{tabular}{lccc}
\hline Variables & Group A $(\mathrm{n}=11)$ & Group B $(\mathrm{n}=17)$ & \multicolumn{1}{c}{ P value } \\
\hline Surgery time $(\mathrm{min})$ & $131.2 \pm 6.7$ & $109.3 \pm 9.7$ & 0.06 \\
Blood loss $(\mathrm{mL})$ & $839.1 \pm 30.6$ & $13.2 \pm 1.7$ & 0.38 \\
Hospital stays $(\mathrm{d})$ & $13.8 \pm 1.6$ & $41.4 \pm 4.8$ & 0.81 \\
Follow-up period (months) & $40.8 \pm 8.4$ & 6 & 0.97 \\
Died & 3 & & 0.58 \\
Complications & & 2 & 1 \\
Wound infection & 3 & 0 & 1 \\
Deep infection & 0 & 0 & 1 \\
Dislocation & 0 & 0 & 0.88 \\
Prosthesis loosening & 0 & 6 & 1 \\
Relapses & 5 & & \\
\hline
\end{tabular}

of hospitalization, but they were cured by daily dressing changes. No patient suffered a deep infection following the procedure. All patients in both groups underwent routine re-testing using standard radiographs at follow-up (X-rays and CT scans). Five patients in group A and six in group $\mathrm{B}$ experienced recurrence. No evidence of periprosthetic fractures, prosthesis loosening or dislocation was found in either group according to the imaging results at most recent follow-up.

In terms of the rehabilitation of limb function, the average postoperative HHS and MSTS at the 24-month follow-up were all higher than those at the preoperative stage, with significant pain relief or functional improvement. At the 6-, 12-, and 24-month follow-up, there were no differences in the HHS or MSTS between the two groups (Table 3).

\section{Discussion}

As a limb salvage reconstruction method, the tumor hip 
Table 3 The follow-up results of HHS and MSTS of all patients

\begin{tabular}{lccc}
\hline Variables & Group A $(n=11)$ & Group B $(n=17)$ & P value \\
\hline HHS & & $66.9 \pm 4.3$ & 0.73 \\
Follow-up 6 months & $67.5 \pm 3.8$ & $80.2 \pm 1.4$ & 0.81 \\
Follow-up 12 months & $79.1 \pm 2.1$ & $84.3 \pm 4.2$ & 0.52 \\
Follow-up 24 months & $85.4 \pm 5.4$ & & 0.48 \\
MSTS & & $16.9 \pm 1.9$ & 0.61 \\
Follow-up 6 months & $17.3 \pm 1.7$ & $23.3 \pm 1.7$ & 0.56 \\
Follow-up 12 months & $23.7 \pm 2.6$ & $25.1 \pm 0.6$ & \\
Follow-up 24 months & $25.3 \pm 0.9$ & & \\
\hline
\end{tabular}

HHS, Harris Hip Score; MSTS, Musculoskeletal Tumor Society Score.

prosthesis offers faster postoperative functional recovery, longer survival time and significantly improved quality of life for patients with bone tumors of the proximal femur through surgical reconstruction after total tumor resection. However, it results in a long segmental bone defect of the proximal femur after extensive resection, which can pose some technical difficulties during the prosthesis implantation procedure, including proper placement of the prosthesis with appropriate anteversion angle. This angle is an essential factor in maintaining stability and decreasing the incidence of dislocation (24). As reported in one study involving 200 adult cadavers, the mean femoral anteversion angle for men is $33.5^{\circ}$ and for women, $12.2^{\circ}$ (25). In total hip arthroplasty (THA) that is not performed for oncological reasons, it is relatively simple to position the components within an appropriate area based on the anatomic landmark of the lesser trochanter. Implanting the femoral stem component based on the dissection shape of the femoral neck fundus and the shape of the medullary canal will result in a position similar to the normal anatomical form. However, if there are developmental abnormalities of the proximal femur such as developmental dysplasia of the hip (DDH) or a significant bone defect caused by oncological reasons, it would likely take additional time to place the components.

In conventional approaches, we recognize the longest axis of cross-section of the medullary cavity after femoral osteotomy first, then pronate at an angle of $5^{\circ}$ and make a mark on the cross section. A new line is then identified based on the mark by visual inspection, the medullary cavity is reamed and then the femoral stem is implanted. At this angle the anteversion angle of the femoral stem would range from $10^{\circ}$ to $20^{\circ}$. The reasonability of this method lies in the fact that the femoral stem's position accords with the normal anatomic morphology of the femoral medullary cavity. It could match the medullary cavity well at this angle according to clinical observation, without causing instability. However, sometimes it can be difficult to determine the longest axis of the medullary cavity and to recognize the typical shape of the medullary cavity after extensive resection that might even cause the angle to become a retroversion angle. Accurate placement of the stem component usually requires repeated adjustment and testing by the conventional method, which will take extra time. Therefore, we continued to explore clinical practices and as a result, propose a modified surgical technique to improve the position of the prosthesis during the implantation procedure.

Figure 5 shows a sagittal reconstruction image of the hip joint, in which the femoral stem anteversion [also called femoral neck anteversion (FNA)] refers to the angle between the axis (line $\mathrm{A}_{2}-\mathrm{H}$ ) of the femoral prosthesis neck and the coronal plane $(\mathrm{H}-\mathrm{K})$ of the femoral condyle; keeping the shin at a 90-degree flexion angle and projecting the posture on this figure, then the line $\mathrm{K}-\mathrm{A}_{1}$ could represent the long axis of the shin. By holding the knee joint, and rotating the ankle joint from point $A_{1}$ to $B_{1}$, the femoral head will consequently move from point $A_{2}$ to $B_{2}$. When the point $B_{2}$ is located on the coronal plane $\left(B_{2}-H-K\right)$, the angle $A_{1} K_{1} B_{1}$ would equate with the femoral stem anteversion. Continuing to rotate the ankle joint from point $B_{1}$ to $D_{1}$ will cause the femoral stem to shift from front angulation to rear angulation. During surgery, the ankle joint is rotated by the estimated amount to make the angle $\mathrm{C}_{1} \mathrm{KD}_{1}$ approximately 


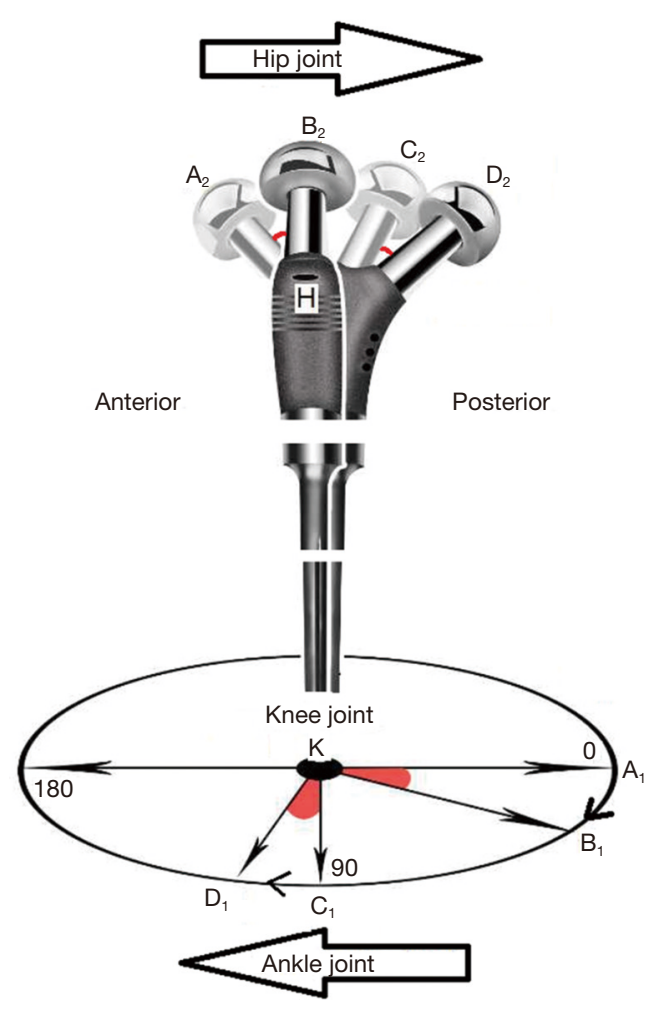

Figure 5 This plane figure shows the linkage mechanism of the hip joint and the hip joint through the knee joint. The anteversion angle of the femoral prosthetic stem will change correspondingly when we rotate the ankle joint by this special procedure.

$15^{\circ}$. At this position, as shown in Figure 3, the medullary cavity is reamed and the femoral components are implanted horizontally, then the femoral head would be in point $\mathrm{D}_{2}$, locating on the sagittal plane. Returning to the normal position after surgery, the anteversion angle of the femoral prosthetic stem will be approximately $15^{\circ}$.

In this study, we retrospectively analyzed the postoperative results of the two groups. The analysis showed that the operative time of group B was significantly less than that of group $\mathrm{A}(\mathrm{P}<0.01)$. From the perspective of a patient undergoing an operation, surgical time means a lot; the longer the duration of an operation the more complications are likely to arise. This modified surgical technique simplifies the procedure of implantation and consequently shortens the surgery time. There were no significant differences in the estimated intraoperative blood loss between the two groups, although it was less in group B than in group A, which may correlate with the shorter operation time. There were no obvious differences between the two groups in hospital stay or postoperative complications. No instances of dislocation, prosthesis loosening, or any other problems related to instability caused by the angle of the femoral stem occurred in the follow-up period in any of the patients. According to the HHS and MSTS scores at the last follow-up, both methods did not affect the functional rehabilitation of the limb. The advantage of this new surgical technique lies in its simplicity and practicality, which could be applied to the treatment of most proximal femoral bone defects caused by oncological reasons.

After extensive tumor resection, endoprosthetic reconstruction could give patients a better quality of life. It is important to place the femoral component accurately, and we conclude that our method can simplify the procedure and shorten the operation time. It is practical and advisable in the treatment of proximal femoral bone defects caused by oncological reasons. We must acknowledge that there are several limitations to this study. First, this was a retrospective study. Secondly, the included sample size is relatively small and therefore lacks statistical significance.

\section{Conclusions}

In summary, the current study described a simple and effective surgical technique for femoral component implantation in the surgery of endoprosthetic reconstruction. This modified surgical technique may be used to improve both the quality and the efficiency of the operation in the treatment of bone tumors of the proximal femur.

\section{Acknowledgments}

Funding: This study was funded by the Natural Science Foundation of China (grant numbers 81871830, 81672236, and 82072524), the Biomedical Translational Engineering Research Center of BUCT-CJFH (grant number RZ202002) and Graduate Innovation Foundation of Peking Union Medical College (grant number 2019-1002-91).

\section{Footnote}

Reporting Checklist: The authors have completed the STROBE reporting checklist. Available at http://dx.doi. org/10.21037/apm-20-895

Data Sharing Statement: Available at http://dx.doi. org/10.21037/apm-20-895 
Conflicts of Interest: All authors have completed the ICMJE uniform disclosure form (available at http://dx.doi. org/10.21037/apm-20-895). The authors have no conflicts of interest to declare.

Ethical Statement: The authors are accountable for all aspects of the work in ensuring that questions related to the accuracy or integrity of any part of the work are appropriately investigated and resolved. All participants gave informed consent prior to participation, and the study conformed to the provisions of the Declaration of Helsinki (as revised in 2013). This study was approved by the Ethics Committee of China-Japan Friendship Hospital (2020QGW-108).

Open Access Statement: This is an Open Access article distributed in accordance with the Creative Commons Attribution-NonCommercial-NoDerivs 4.0 International License (CC BY-NC-ND 4.0), which permits the noncommercial replication and distribution of the article with the strict proviso that no changes or edits are made and the original work is properly cited (including links to both the formal publication through the relevant DOI and the license). See: https://creativecommons.org/licenses/by-nc-nd/4.0/.

\section{References}

1. Hage WD, Aboulafia AJ, Aboulafia DM. Incidence, location, and diagnostic evaluation of metastatic bone disease. Orthop Clin North Am 2000;31:515-28.

2. Cardelia JM, Dormans JP, Drummond DS, et al. Proximal fibular osteochondroma with associated peroneal nerve palsy: a review of six cases. J Pediatr Orthop 1995;15:574-7.

3. Barros Filho TE, Oliveira RP, Taricco MA, et al. Hereditary multiple exostoses and cervical ventral protuberance causing dysphagia. A case report. Spine (Phila Pa 1976) 1995;20:1640-2.

4. Hunt KJ, Gollogly S, Randall RL. Surgical fixation of pathologic fractures: an evaluation of evolving treatment methods. Bull Hosp Jt Dis 2006;63:77-82.

5. Ward WG, Holsenbeck S, Dorey FJ, et al. Metastatic disease of the femur: surgical treatment. Clin Orthop Relat Res 2003;415:S230-44.

6. Samsani SR, Panikkar V, Venu KM, et al. Breast cancer bone metastasis in femur: surgical considerations and reconstruction with Long Gamma Nail. Eur J Surg Oncol 2004;30:993-7.

7. van Doorn R, Stapert JW. Treatment of impending and actual pathological femoral fractures with the long Gamma nail in The Netherlands. Eur J Surg 2000;166:247-54.

8. Kabukcuoglu Y, Grimer RJ, Tillman RM, et al. Endoprosthetic replacement for primary malignant tumors of the proximal femur. Clin Orthop Relat Res 1999;358:8-14.

9. Dobbs HS, Scales JT, Wilson JN, et al. Endoprosthetic replacement of the proximal femur and acetabulum. A survival analysis. J Bone Joint Surg Br 1981;63-B:219-24.

10. Doung YC, Kenan S, Rapp T. Metastatic lesions of the proximal femur. Bull NYU Hosp Jt Dis 2011;69:81-6.

11. Brien EW, Terek RM, Healey JH, et al. Allograft reconstruction after proximal tibial resection for bone tumors. An analysis of function and outcome comparing allograft and prosthetic reconstructions. Clin Orthop Relat Res 1994;303:116-27.

12. Wirganowicz PZ, Eckardt JJ, Dorey FJ, et al. Etiology and results of tumor endoprosthesis revision surgery in 64 patients. Clin Orthop Relat Res 1999;358:64-74.

13. Zeegen EN, Aponte-Tinao LA, Hornicek FJ, et al. Survivorship analysis of 141 modular metallic endoprostheses at early followup. Clin Orthop Relat Res 2004;420:239-50.

14. Moon BS, Gilbert NF, Cannon CP, et al. Distal femur allograft prosthetic composite reconstruction for short proximal femur segments following tumor resection. Adv Orthop 2013;2013:397456.

15. Benedetti MG, Bonatti E, Malfitano, et al. Comparison of allograft-prosthetic composite reconstruction and modular prosthetic replacement in proximal femur bone tumors: functional assessment by gait analysis in 20 patients. Acta Orthop 2013;84:218-23.

16. Henderson ER, Groundland JS, Pala E, et al. Failure mode classification for tumor endoprostheses: retrospective review of five institutions and a literature review. J Bone Joint Surg Am 2011;93:418-29.

17. Bernthal NM, Schwartz AJ, Oakes DA, et al. How long do endoprosthetic reconstructions for proximal femoral tumors last? Clin Orthop Relat Res 2010;468:2867-74.

18. Schneiderbauer MM, Sierra RJ, Schleck C, et al. Dislocation rate after hip hemiarthroplasty in patients with tumor-related conditions. J Bone Joint Surg Am 2005;87:1810-5.

19. Herrlin K, Pettersson H, Selvik G, et al. Femoral anteversion and restricted range of motion in total hip prostheses. Acta Radiol 1988;29:551-3.

20. Weiner DS, Cook AJ, Hoyt WA, et al. Computed tomography in the measurement of femoral anteversion. 
Orthopedics 1978;1:299-306.

21. Biermann JS, Adkins DR, Benjamin RS, et al. Bone Cancer, Version 3.2010, NCCN Clinical Practice Guidelines in Oncology. J Natl Compr Canc Netw 2010;8:688-712.

22. Harris WH. Traumatic arthritis of the hip after dislocation and acetabular fractures. treatment by mold arthroplasty. An end-result study using a new method of result evaluation. J Bone Joint Surg Am 1969;51:737-55.

23. Enneking WF, Dunham W, Gebhardt MC, et al. A system for the functional evaluation of reconstructive procedures after surgical treatment of tumors of the musculoskeletal system. Clin Orthop Relat Res 1993;286:241-6.

24. Barrack RL. Dislocation after total hip arthroplasty: implant design and orientation. J Am Acad Orthop Surg 2003;11:89-99.

25. Maruyama M, Feinberg JR, Capello WN, et al. The Frank Stinchfield Award: Morphologic features of the acetabulum and femur: anteversion angle and implant positioning. Clin Orthop Relat Res 2001;393:52-65.
Cite this article as: Shi L, Han J, Shi L, Sun W, Gao F. A modified surgical technique to simplify the procedure of femoral stem implantation for an appropriate anteversion angle during endoprosthetic reconstruction. Ann Palliat Med 2021;10(2):1620-1630. doi: 10.21037/apm-20-895 